\title{
Gauge Theory on Twisted $\kappa$-Minkowski: Old Problems and Possible Solutions*
}

\author{
Marija DIMITRIJEVIĆ $\dagger$, Larisa JONKE $\ddagger$ and Anna PACHOE $§$ \\ $\dagger$ University of Belgrade, Faculty of Physics, Studentski trg 12, 11000 Beograd, Serbia \\ E-mail:dmarija@ipb.ac.rs \\ ¥Division of Theoretical Physics, Rudjer Bošković Institute, Bijenička 54, 10000 Zagreb, Croatia \\ E-mail: larisa@irb.hr \\ $\S$ Science Institute, University of Iceland, Dunhaga 3, $10^{77}$ Reykjavik, Iceland \\ E-mail: pachol@hi.is
}

Received March 10, 2014, in final form June 05, 2014; Published online June 14, 2014

http://dx.doi.org/10.3842/SIGMA.2014.063

\begin{abstract}
We review the application of twist deformation formalism and the construction of noncommutative gauge theory on $\kappa$-Minkowski space-time. We compare two different types of twists: the Abelian and the Jordanian one. In each case we provide the twisted differential calculus and consider $U(1)$ gauge theory. Different methods of obtaining a gauge invariant action and related problems are thoroughly discussed.
\end{abstract}

Key words: $\kappa$-Minkowski; twist; $U(1)$ gauge theory; Hodge dual

2010 Mathematics Subject Classification: 81T75; 58B22

\section{Introduction}

Noncommutative (NC) deformations of space-time, introduced either through effective models or as fundamental property of a theory, lead to models that can mimic some of the properties expected in a quantized theory of gravity. In particular, the combination of general relativity and quantum mechanics suggests that at the Plank scale the standard picture of space-time as a smooth manifold breaks down and should be replaced by some kind of fuzzy or foam-like spacetime. This property can be naturally realized by NC deformations of space-time, with prominent examples being fuzzy spaces [27, 39, 28] and, more generally, matrix models [14, 51, 13]. Furthermore, it is expected that our understanding and implementation of symmetries should be modified when describing physics close to the Planck scale. Disentangling the geometry from matter content of a theory at this scale is non-trivial, and mixing of internal/gauge symmetries and geometry/diffeomorphisms occurs. In construction of a physical theory on NC space-time one implements this property by introducing noncommutative gauge transformations [31, 30].

The construction of NC gauge theory is an important step in understanding the physics on NC space-time. Indeed, the main concept in quantum field theory underlying the success of the Standard model is the principle of local gauge symmetries. Therefore an important aspect of the construction of the gauge theory on $\mathrm{NC}$ spaces is a consistent implementation of local gauge symmetry in combination with non-locality introduced implicitly through a NC deformation of space-time. A key ingredient in such construction is the Seiberg-Witten map [49]. This mapping includes a set of non-local and non-linear field redefinitions relating commutative and NC gauge fields and parameters. Most importantly, it enables a consistent definition of NC gauge theories for arbitrary gauge groups.

${ }^{\star}$ This paper is a contribution to the Special Issue on Deformations of Space-Time and its Symmetries. The full collection is available at http://www.emis.de/journals/SIGMA/space-time.html 
In describing NC space-time, we substitute the concept of manifold with an algebra of functions on manifold. NC deformations of such algebra correspond to NC deformations of spacetime. There exist powerful methods for studying deformations of an algebra of functions if this algebra carries a representation of a Hopf algebra. In this case one first considers a deformation of the Hopf algebra, and then uses the Hopf algebra action in order to induce a deformation of the algebra on functions on manifold. Deformation via Drinfel'd twists [21] is an example of such procedure. One of the main advantages of this formalism is the straightforward way to define a differential calculus, an important ingredient in the construction of a (gauge) field theory.

In this work our primary interest is to examine compatibility of the local gauge principle with the deformation of algebra of functions on a specific example of NC space-time, the $\kappa$ Minkowski space-time. This example of noncommutative space is the most analysed example of non-constant deformation with potentially interesting phenomenological consequences [35]. The (non-trivial) commutation relations of coordinates of the four-dimensional $\kappa$-Minkowski spacetime are of the Lie-algebra type $\left[x^{0}, x^{j}\right]=i \kappa^{-1} x^{j}$, where $j$ denotes the space directions and the zeroth component corresponds to the time direction. One of the important properties of this NC space-time is that there is a quantum group symmetry acting on it. It is a dimensionfull deformation of the global Poincaré group, the $\kappa$-Poincaré group [38, 37]. The constant $\kappa$ has dimension of energy and sets a deformation scale. The construction of field theory on this Lie algebraic type of noncommutative spacetime attracted a lot of interest, but was mainly concentrated on the scalar field theories [19, 1, 15, 48, 42]. The problem of constructing gauge field theory on $\kappa$-Minkowski space-time was addressed in [20, 18].

All these results have shown that the construction of field theories on this space is plagued with ambiguities, mostly due to the lack of understanding of the symmetries of NC space. We proposed to resolve some of these ambiguities by using specific Abelian twist to introduce deformation in the algebra $[16,17]$. We constructed the action for the noncommutative $U(1)$ gauge fields in a geometric way, as an integral of a maximal form. However, we could not maintain the $\kappa$-Poincaré symmetry; the corresponding symmetry of the twisted $\kappa$-Minkowski space was the twisted $\operatorname{igl}(1,3)$ symmetry. It turned out that this is a generic situation; $\kappa$ Minkowski space-time obtained from $\kappa$-Poincaré algebra cannot be obtained by twisting the usual Poincaré algebra of symmetry [9]. Still, the extensions of Poincaré algebra are amenable to twist formulation, and in this work we concentrate on two particular examples. The twists with support in extensions of Poincaré algebra were also considered previously in $[6,7,11,26]$.

In Sections 2 and 3 we review the basics of the twist formalism and the Seiberg-Witten map; two main concepts we use in our analysis. Then, in Sections 4 and 5 we introduce two different twists (Abelian and Jordanian). Commutation relations of coordinates that follow from those twists are commutation relations of the $\kappa$-Minkowski space-time; the twisted symmetry algebra is not the $\kappa$-Poincaré algebra. We construct the twisted differential calculus for both deformations. Finally, we discuss the $U(1)$ gauge theory obtained from two different twists. We describe the problems related to the integration and the construction of the Hodge dual in details and give some possible solutions. We extend the analysis of the deformed gauge theory based on the Abelian twist $[16,17]$ and compare it with the one based on the Jordanian twist. We explicitly show how the difference in the underlying twisted symmetry is manifested at the level of gauge theory action.

\section{Twist formalism}

Within the twist formalism the NC deformations are introduced by twisting the underlying symmetry of the theory and then consistently applying the consequences of the deformation on the geometry of space-time itself. The underlying symmetries are described in the Hopf algebra language and the $\mathrm{NC}$ spaces (as deformed algebras of functions) are Hopf module algebras. 
The twisted symmetry does not have the usual dynamical significance and, in particular, there is no Noether procedure associated with it. We view this symmetry as a prescription that allows us to consistently apply deformation in the theory. The twist deformation equips the algebra of smooth compactly supported functions $A=C_{c}^{\infty}(M)$ with the twisted $\star$-product and can be represented by deformed, $\star$-commutators of noncommutative coordinates ${ }^{1}$. Since we are interested in deformations of space-time symmetries, we concentrate on the Lie algebra of vector fields and its deformations; generalization to any Lie algebra is straightforward.

Vector fields provide an infinite dimensional Lie algebra, its universal enveloping algebra includes linear differential operators. Those act naturally on the algebra of functions (Hopf module algebra) on a manifold. We work in four dimensions and use the Lorentzian signature (mostly minus). Generalization to $n$ dimensions is easily done. The Lie algebra of vector fields is denoted by $\Xi$ and its elements are vector fields $\xi$, which can be written in the coordinate basis as: $\xi=\xi^{\mu} \frac{\partial}{\partial x^{\mu}}=\xi^{\mu} \partial_{\mu}$. This algebra generates the diffeomorphism symmetry; one can also consider subgroups of $\Xi$ like Poincaré algebra or conformal algebra as symmetry groups. The universal enveloping algebra of $\Xi$ we denote by $U \Xi$. It can be equipped with the Hopf algebra structure:

$$
\begin{aligned}
& {[\xi, \eta]=\left(\xi^{\mu} \partial_{\mu} \eta^{\rho}-\eta^{\mu} \partial_{\mu} \xi^{\rho}\right) \partial_{\rho},} \\
& \Delta(\xi)=\xi \otimes 1+1 \otimes \xi, \\
& \varepsilon(\xi)=0, \quad S(\xi)=-\xi .
\end{aligned}
$$

The first line is the algebra relation: commutator of two vector fields is a vector field. In the second line the coproduct of the generator $\xi$ is given; note that it is primitive. It encodes the Leibniz rule and specifies how the symmetry transformation acts on products of fields/representations. In the last line, the counit and the antipode maps are given.

A well defined way to deform the symmetry Hopf algebra is via twist. The twist $\mathcal{F}$ is an invertible element of $U \Xi \otimes U \Xi$ satisfying the following properties:

1) the cocycle condition

$$
(\mathcal{F} \otimes 1)(\Delta \otimes \mathrm{id}) \mathcal{F}=(1 \otimes \mathcal{F})(\mathrm{id} \otimes \Delta) \mathcal{F}
$$

2) normalization

$$
(\mathrm{id} \otimes \epsilon) \mathcal{F}=(\epsilon \otimes \mathrm{id}) \mathcal{F}=1 \otimes 1,
$$

3) perturbative expansion

$$
\mathcal{F}=1 \otimes 1+\mathcal{O}(\lambda)
$$

where $\lambda$ is a deformation parameter. The last property provides an undeformed case at the zeroth order in parameter $\lambda$. We shall frequently use the notation (sum over $\alpha=1,2, \ldots, \infty$ is understood)

$$
\mathcal{F}=\mathrm{f}^{\alpha} \otimes \mathrm{f}_{\alpha}, \quad \mathcal{F}^{-1}=\overline{\mathrm{f}}^{\alpha} \otimes \overline{\mathrm{f}}_{\alpha},
$$

where, for each value of $\alpha, \overline{\mathrm{f}}^{\alpha}$ and $\overline{\mathrm{f}}_{\alpha}$ are two distinct elements of $U \Xi$ (and similarly $\mathrm{f}^{\alpha}$ and $\mathrm{f}_{\alpha}$ are in $U \Xi)^{2}$. The twist acts on the symmetry Hopf algebra and gives the twisted symmetry (as

\footnotetext{
${ }^{1}$ Throughout this paper differential forms are smooth and compactly supported.

${ }^{2}$ Strictly speaking a twisted deformation of the Lie algebra $\Xi$ requires a topological extension of the corresponding enveloping algebra $U \Xi$ into an algebra of formal power series $U \Xi[[\lambda]]$ in the formal parameter $\lambda$. Then the twisting element, can be rewritten as a power series expansion $\mathcal{F}=1 \otimes 1+\sum_{\alpha=1}^{\infty} \lambda^{\alpha} \mathrm{f}^{\alpha} \otimes \mathrm{f}_{\alpha}$.
} 
deformed Hopf algebra)

$$
\begin{aligned}
& {[\xi, \eta]=\left(\xi^{\mu} \partial_{\mu} \eta^{\rho}-\eta^{\mu} \partial_{\mu} \xi^{\rho}\right) \partial_{\rho},} \\
& \Delta^{\mathcal{F}}(\xi)=\mathcal{F} \Delta(\xi) \mathcal{F}^{-1} \\
& \varepsilon\left(t^{a}\right)=0, \quad S^{\mathcal{F}}(\xi)=\mathrm{f}^{\alpha} S\left(\mathrm{f}_{\alpha}\right) S(\xi) S\left(\overline{\mathrm{f}}^{\beta}\right) \overline{\mathrm{f}}_{\beta} .
\end{aligned}
$$

The algebra remains the same, while in general the comultiplication (coproduct) and antipode change. The whole deformation depends on formal parameters which control classical limit. Twisted (deformed) comultiplication leads to the deformed Leibniz rule for the symmetry transformations when acting on product of fields.

Now we use the twist to deform the commutative geometry of space-time (functions, vector fields, exterior algebra of forms, tensor fields). As usual in physics, we will work with smooth complex tensor fields. In particular, we work with smooth functions, smooth exterior algebra of forms, smooth vector fields. For more details see for example Chapter 1 in [47]. We consider one particular class of twists, the Abelian twists [5, 29]

$$
\mathcal{F}=e^{-\frac{i}{2} \theta^{C D} X_{C} \otimes X_{D}} .
$$

Here $\theta^{C D}$ is a constant antisymmetric matrix, $C, D=1, \ldots, p, p \leq 4$ and $X_{C}=X_{C}^{\mu} \partial_{\mu}$ are commuting vector fields, $\left[X_{C}, X_{D}\right]=0$. This twist fulfils the requirements $(2.2)-(2.4)$. We write all results for this particular twist, but we mention if and when they are valid in more general cases (more general twists).

Applying the inverse of the twist (2.7) to the usual point-wise multiplication of functions, $\mu(f \otimes g)=f \cdot g ; f, g \in A$, we obtain the $\star$-product of functions

$$
f \star g=\mu \mathcal{F}^{-1}(f \otimes g)=\overline{\mathrm{f}}^{\alpha}(f) \overline{\mathrm{f}}_{\alpha}(g) .
$$

The action of the twist $\left(\overline{\mathrm{f}}^{\alpha}\right.$ and $\left.\overline{\mathrm{f}}_{\alpha}\right)$ on the functions $f$ and $g$ is via the Lie derivative. This «-product is noncommutative, associative and in the limit $\theta^{C D} \rightarrow 0$ it reduces to the usual pointwise multiplication; the last property is guaranteed by (2.4) and the associativity is guaranteed by (2.2). In this way we obtain the noncommutative algebra of functions $A^{\mathcal{F}}=(A, \star)$, i.e. the noncommutative space-time. The product between functions and 1-forms is defined as

$$
h \star \omega=\overline{\mathrm{f}}^{\alpha}(h) \overline{\mathrm{f}}_{\alpha}(\omega)
$$

with an arbitrary 1-form $\omega$. The action of $\overline{\mathrm{f}}_{\alpha}$ on forms is (again) given via the Lie derivative. We often use the Cartan's formula for the Lie derivative along the vector field $\xi$ of an arbitrary form $\omega$

$$
l_{\xi} \omega=\mathrm{d} i_{\xi} \omega+i_{\xi} \mathrm{d} \omega .
$$

Here $\mathrm{d}$ is the exterior derivative and $i_{\xi}$ is the contraction along the vector field $\xi$.

Arbitrary forms form an exterior algebra with the wedge product. The $\star$-wedge product on two arbitrary forms $\omega$ and $\omega^{\prime}$ is

$$
\omega \wedge_{\star} \omega^{\prime}=\overline{\mathrm{f}}^{\alpha}(\omega) \wedge \overline{\mathrm{f}}_{\alpha}\left(\omega^{\prime}\right) .
$$

The usual (commutative) exterior derivative $\mathrm{d}: A \rightarrow \Omega$ satisfies:

$$
\begin{aligned}
& \mathrm{d}(f \star g)=\mathrm{d} f \star g+f \star \mathrm{d} g, \quad \mathrm{~d}^{2}=0, \\
& \mathrm{~d} f=\left(\partial_{\mu} f\right) \mathrm{d} x^{\mu}=\left(\partial_{\mu}^{\star} f\right) \star \mathrm{d} x^{\mu} .
\end{aligned}
$$

The first property if fulfilled because the usual exterior derivative commutes with the Lie derivative which enters in the definition of the $\star$-product. Therefore, we will use the usual exterior 
derivative as the noncommutative exterior derivative. Note that the last line of (2.8) gives a definition of the $\partial_{\mu}^{\star}$ derivatives.

All the properties and definitions introduced so far are also valid for a more general twist. However with the definition of integral one has to be more careful. The usual integral is cyclic under the $\star$-exterior products of forms

$$
\int \omega_{1} \wedge_{\star} \omega_{2}=(-1)^{d_{1} \cdot d_{2}} \int \omega_{2} \wedge_{\star} \omega_{1},
$$

where $d=\operatorname{deg}(\omega), d_{1}+d_{2}=4$ provided that $S\left(\overline{\mathrm{f}}^{\alpha}\right) \overline{\mathrm{f}}_{\alpha}=1$ holds, for more details see [2]. One can check that this indeed holds for the Abelian twist (2.7).

Note that this approach to deformation of differential calculus, i.e. twisted approach differs from the bicovariant differential calculi formulation. More specifically, the covariance condition, i.e. $L \triangleright(f g)=\left(L_{(1)} \triangleright f\right)\left(L_{(2)} \triangleright g\right), L \in U \Xi, f, g \in A$ is satisfied in both approaches (the NC space-time is a Hopf module algebra). However the bicovariance condition (requiring that all $\mathrm{d} x_{\mu}$, must be simultaneously left and right-invariant) is not satisfied in the twisted version. Moreover it has been shown that in the case of $\kappa$-Minkowski space-time the four-dimensional bicovariant differential calculi does not exist, but one can construct a five-dimensional one, which is bicovariant [50]. Alternative approaches to differential calculus on $\kappa$-Minkowski space-time were also considered in [34, 41, 33].

\section{NC gauge theory and the Seiberg-Witten map}

In this section we describe how to construct a $\mathrm{NC}$ gauge theory on a deformed space-time obtained from the Abelian twist (2.7). To achieve our goal we use the enveloping algebra approach and the Seiberg-Witten map, as developed in [31,30]. To have more general results, we work with an arbitrary Lie group $G$, with generators $T^{a}$ and $\left[T^{a}, T^{b}\right]=i f^{a b c} T^{c}$. The obtained results are then easy to specify to the case of $U(1)$ gauge group. Noncommutative fields we label with a "hat" and commutative without a "hat". Under the infinitesimal NC gauge transformations the NC gauge field ${ }^{3} \hat{A}=\hat{A}_{\mu} \star \mathrm{d} x^{\mu}$ transforms as

$$
\delta_{\alpha}^{\star} \hat{A}=\mathrm{d} \hat{\Lambda}_{\alpha}+i\left[\hat{\Lambda}_{\alpha}, \hat{A}\right],
$$

with the NC gauge parameter $\hat{\Lambda}_{\alpha}$. The NC gauge parameter is a noncommutative function valued (as we shall see later) in the enveloping algebra of the gauge group. As all NC functions, it is represented by a function of the commuting coordinates and it is a power series expansion in the deformation parameter. The index $\alpha$ signals that in the zeroth order of the deformation parameter the NC gauge parameter $\hat{\Lambda}_{\alpha}$ reduces to the commutative, Lie algebra-valued gauge parameter $\alpha=\alpha^{a} T^{a}$. Note that this index $\alpha$ is not related with the index $\alpha$ in Section 2, equation (2.5). We demand that the consistency condition is satisfied, i.e. transformations (3.1) have to close the algebra

$$
\left[\delta_{\alpha}^{\star}, \delta_{\beta}^{\star}\right]=\delta_{-i[\alpha, \beta]}^{\star} \cdot
$$

This will be the case provided that the gauge parameter $\hat{\Lambda}_{\alpha}$ is in the enveloping algebra of the algebra $g$ of the gauge group $G .{ }^{4}$ However, an enveloping algebra is infinite dimensional and the

\footnotetext{
${ }^{3}$ Note that we can expand the noncommutative forms in the coordinate basis in two different ways $\omega=$ $\omega_{\mu} \star \mathrm{d} x^{\mu}=\tilde{\omega}_{\mu} \mathrm{d} x^{\mu}$. The difference will only be in the components of forms. Depending on the situation, we will use one or the other expansion, but we will be careful not to mix them.

${ }^{4}$ Note that in (3.1) $\star$-commutators appear. These commutators do not close in the Lie algebra, namely having $A=A^{a} T^{a}$ and $B=B^{a} T^{a}$ leads to$$
[A \star B]=\frac{1}{2}\left(A^{a} \star B^{b}+B^{b} \star A^{a}\right)\left[T^{a}, T^{b}\right]+\frac{1}{2}\left(A^{a} \star B^{b}-B^{b} \star A^{a}\right)\left\{T^{a}, T^{b}\right\} .
$$ 
resulting theory seems to have infinitely many degrees of freedom. This problem is solved by the Seiberg-Witten map. The idea of the Seiberg-Witten map is that all noncommutative variables (gauge parameter, fields) can be expressed in terms of the corresponding commutative variables and their derivatives; then the $\mathrm{NC}$ gauge transformations are induced by the corresponding commutative gauge transformations

$$
\hat{A}(A)+\delta_{\alpha}^{\star} \hat{A}(A)=\hat{A}\left(A+\delta_{\alpha} A\right),
$$

with the commutative gauge field $A=A^{a} T^{a}$ and the commutative gauge parameter $\alpha=\alpha^{a} T^{a}$. In addition, we assume that we can expand all $\mathrm{NC}$ variables as power series in noncommutativity parameter $\theta^{C D}$ introduced by the twist.

In the case of $\mathrm{NC}$ gauge parameter the expansion is

$$
\hat{\Lambda}_{\alpha}=\Lambda_{\alpha}^{(0)}+\Lambda_{\alpha}^{(1)}+\Lambda_{\alpha}^{(2)}+\cdots,
$$

with $\Lambda_{\alpha}^{(0)}=\alpha$. Inserting this expansion into (3.2) and expanding all $\star$-products gives a variational equation for the gauge parameter $\hat{\Lambda}_{\alpha}$. This equation can be solved to all orders of the deformation parameter. The zeroth order solution is the commutative gauge parameter $\alpha$. The recursive relation between the $n$th and the $(n+1)$ st order solution is given by $[53,3]$

$$
\hat{\Lambda}_{\alpha}^{(n+1)}=-\frac{1}{4(n+1)} \theta^{C D}\left\{\hat{A}_{C}{ }^{\star}, l_{D} \hat{\Lambda}_{\alpha}\right\}^{(n)},
$$

where $(A \star B)^{(n)}=A^{(n)} B^{(0)}+A^{(n-1)} B^{(1)}+\cdots+A^{(0)} \star^{(1)} B^{(n-1)}+A^{(1)} \star^{(1)} B^{(n-2)}+\cdots$ includes all possible terms of order $n$. We introduced the following notation: $\hat{A}_{C}=i_{X_{C}} \hat{A}$ is a contraction of the 1-form $\hat{A}$ along the vector field $X_{C}$ and $l_{D}$ is a Lie derivative along the vector field $X_{D}$.

Solving the equation (3.3) order by order in the NC parameter the NC gauge field $\hat{A}$ is expressed in terms of the commutative gauge field $A$. The recursive solution in this case is given by

$$
\hat{A}^{(n+1)}=-\frac{1}{4(n+1)} \theta^{C D}\left\{\hat{A}_{C}{ }^{\star} l_{D} \hat{A}+\hat{F}_{D}\right\}^{(n)},
$$

where $L_{D} \hat{A}=l_{D} \hat{A}-i\left[\hat{A}_{D}, \hat{A}\right]$ and $\hat{F}_{D}=i_{X_{D}} \hat{F}$.

Finally, the field-strength tensor is defined as $\hat{F}=\mathrm{d} \hat{A}-i \hat{A} \wedge_{\star} \hat{A}$ and it transforms covariantly under infinitesimal NC gauge transformations,

$$
\delta_{\alpha}^{\star} \hat{F}=i[\hat{\Lambda} \stackrel{\star}{*}] .
$$

The recursive relation for the SW map solution is given by

$$
\hat{F}^{(n+1)}=-\frac{1}{4(n+1)} \theta^{C D}\left(\left\{\hat{A}_{C}^{\star},\left(l_{D}+L_{D}\right) \hat{F}\right\}^{(n)}-\left[\hat{F}_{C} \stackrel{\star}{,} \hat{F}_{D}\right]^{(n)}\right),
$$

with the 1 -form $\hat{F}_{C}=i_{X_{C}} \hat{F}$ and the 2 -form $L_{C} \hat{F}=l_{C} \hat{F}-i\left[\hat{A}_{C} \star \hat{F}\right]$. Also, $\left[\hat{F}_{C} \stackrel{*}{*} \hat{F}_{D}\right]=$ $\hat{F}_{C} \wedge_{\star} \hat{F}_{D}-\hat{F}_{D} \wedge_{\star} \hat{F}_{C}$.

Remark 1. The above SW map solutions are written in the language of forms and with the use of the recursive relations. One can also expand these relations in orders of the deformation parameter $\theta^{C D}$ and write the solutions for the components. These will depend on the particular form of the twist as we will see later on. In Sections 4 and 5, we discuss particular examples of the twisted $\kappa$-Minkowski. There we will write the component expansions and we will write them up to first order in the NC parameter.

Remark 2. The recursive solutions are valid for the Abelian twist. For a more general twist one has to solve the SW map order by order in parameter expansion.

Only in the case of $U(n)$ in the defining representation the anticommutator of generators is still in the corresponding Lie algebra. 


\section{Kappa-Minkowski from an Abelian twist}

The main object of this review is the $\kappa$-Minkowski space-time. We will discuss two different ways of twisting that result in $\kappa$-Minkowski space-time. The starting point in both approaches is the symmetry algebra of the four dimensional Minkowski space-time, the Poincaré algebra iso $(1,3)$. It has 10 generators: 4 generators of translations $P_{\mu}$ and 6 generators of Lorentz rotations $M_{\mu \nu}$. The algebra relations are ${ }^{5}$

$$
\begin{aligned}
& {\left[P_{\mu}, P_{\nu}\right]=0, \quad\left[M_{\mu \nu}, P_{\rho}\right]=\eta_{\nu \rho} P_{\mu}-\eta_{\mu \rho} P_{\nu},} \\
& {\left[M_{\mu \nu}, M_{\rho \sigma}\right]=\eta_{\mu \sigma} M_{\nu \rho}+\eta_{\nu \rho} M_{\mu \sigma}-\eta_{\mu \rho} M_{\nu \sigma}-\eta_{\nu \sigma} M_{\mu \rho},}
\end{aligned}
$$

with $\eta_{\mu \nu}=\operatorname{diag}(+1,-1,-1,-1)$. The universal enveloping algebra of this algebra we label with Uiso(1,3). Besides the algebra relations $(4.1)$ Uiso(1,3) can be equipped with the additional structure

$$
\begin{aligned}
& \Delta P_{\mu}=P_{\mu} \otimes 1+1 \otimes P_{\mu}, \quad \varepsilon\left(P_{\mu}\right)=0, \quad S\left(P_{\mu}\right)=-P_{\mu} \\
& \Delta M_{\mu \nu}=M_{\mu \nu} \otimes 1+1 \otimes M_{\mu \nu}, \quad \varepsilon\left(M_{\mu \nu}\right)=0, \quad S\left(M_{\mu \nu}\right)=-M_{\mu \nu} .
\end{aligned}
$$

It is the Hopf algebra we want to deform via twist. Unfortunately, we cannot choose a twist from $\operatorname{Uiso}(1,3) \otimes U i s o(1,3)$ and obtain the $\kappa$-Minkowski space-time in the same time [9]. It follows from the fact that the $\kappa$-deformation of Poincaré algebra is characterized by a classical r-matrix which satisfies inhomogeneous Yang-Baxter equation and one can not obtain the $\kappa$ Poincaré Hopf algebra and $\kappa$-Minkowski as its module from an internal twist. Therefore, in order to obtain the $\kappa$-Minkowski space-time by twisting, we have to enlarge the starting symmetry algebra.

In our first example we choose an Abelian twist given by

$$
\mathcal{F}=e^{-\frac{i}{2} \theta^{C D} X_{C} \otimes X_{D}}=e^{-\frac{i a}{2}\left(\partial_{0} \otimes x^{j} \partial_{j}-x^{j} \partial_{j} \otimes \partial_{0}\right)},
$$

with two commuting vector fields $X_{1}=\partial_{0}$ and $X_{2}=x^{j} \partial_{j}$ and indices $j=1,2,3$. The constant matrix $\theta^{C D}$ is defined as

$$
\theta^{C D}=\left(\begin{array}{cc}
0 & a \\
-a & 0
\end{array}\right)
$$

This twist fulfils the conditions (2.2), (2.3) and (2.4) with the small deformation parameter $\lambda=a$. Detailed analysis of the consequences of this twist and the construction of the $U(1)$ gauge theory was done in $[16,17]$. Therefore, we skip some details here and describe the main problems and results.

The vector field $X_{1}=\partial_{0}$ generates translations along $x^{0}$ and belongs to the Poincaré algebra iso(1,3). However, the vector field $X_{2}=x^{j} \partial_{j}$ belongs to the general linear algebra $g l(1,3)$. Therefore, we have to consider the inhomogeneous general linear algebra $i g l(1,3)$ as our starting point for the symmetry analysis and the twist (4.2) then defines $U_{i g l(1,3)}^{\mathcal{F}}[[a]]$. The commutation relations of $i g l(1,3)$ are

$$
\left[L_{\mu \nu}, L_{\rho \sigma}\right]=\eta_{\nu \rho} L_{\mu \sigma}-\eta_{\mu \sigma} L_{\rho \nu}, \quad\left[P_{\mu}, P_{\nu}\right]=0, \quad\left[L_{\mu \nu}, P_{\rho}\right]=-\eta_{\mu \rho} P_{\nu}
$$

The action of the twist (4.2) on the $\operatorname{igl}(1,3)$ algebra follows from (2.6) and it has been analysed in detail in [10]. The most important results are: the algebra (4.3) remains the same; since $X_{2}=x^{j} \partial_{j}$ does not commute with the generators $P_{\mu}$ and $L_{\mu \nu}$ the comultiplication and the

\footnotetext{
${ }^{5}$ We are working with anti-hermitean generators.
} 
antipode change. In this way we obtain the twisted $\operatorname{igl}(1,3)$ Hopf algebra instead of the $\kappa$ Poincaré algebra found in [38, 37].

The Lie algebra $\operatorname{igl}(1,3)$ contains Poincaré algebra $i s o(1,3)$ as a subalgebra where the Lorentz generators $M_{\mu \nu}$ are defined by: $M_{\mu \nu}=L_{\mu \nu}-L_{\nu \mu}$. The algebra $i g l(1,3)$, as well as its classical subalgebras act on the algebra of functions $A$ via first order differential operators, i.e. vector fields, defined by the natural representation $L_{\mu \nu}=x_{\mu} \partial_{\nu}, P_{\mu}=\partial_{\mu}$. The inverse of the twist (4.2) defines the $\star$-product between functions (fields) on the $\kappa$-Minkowski space-time

$$
\begin{aligned}
f \star g & =\mu\left\{\mathcal{F}^{-1} f \otimes g\right\}=\mu\left\{e^{\frac{i a}{2}\left(\partial_{0} \otimes x^{j} \partial_{j}-x^{j} \partial_{j} \otimes \partial_{0}\right)} f \otimes g\right\} \\
& =f \cdot g+\frac{i a}{2} x^{j}\left(\left(\partial_{0} f\right) \partial_{j} g-\left(\partial_{j} f\right) \partial_{0} g\right)+\mathcal{O}\left(a^{2}\right) \\
& =f \cdot g+\frac{i}{2} C_{\lambda}^{\rho \sigma} x^{\lambda}\left(\partial_{\rho} f\right) \cdot\left(\partial_{\sigma} g\right)+\mathcal{O}\left(a^{2}\right),
\end{aligned}
$$

with $C_{\lambda}^{\rho \sigma}=a\left(\delta_{0}^{\rho} \delta_{\lambda}^{\sigma}-\delta_{0}^{\sigma} \delta_{\lambda}^{\rho}\right)$. This product is associative, noncommutative and hermitean

$$
\overline{f \star g}=\bar{g} \star \bar{f} .
$$

The usual complex conjugation we label with "bar". In the zeroth order (4.4) reduces to the usual point-wise multiplication. Calculating the commutation relations between the coordinates we obtain

$$
\left[x^{0}, x^{j}\right]=x^{0} \star x^{j}-x^{j} \star x^{0}=i a x^{j}, \quad\left[x^{i} \star x^{j}\right]=0 .
$$

These are the commutation relations of the $\kappa$-Minkowski space-time with $a=\kappa^{-1}$.

\subsection{Twisted differential calculus and integration}

We have seen in Section 2 that the NC exterior derivative is the usual exterior derivative with the properties (2.8). We now discuss the specific properties due to the twist (4.2).

The basis 1-forms are $\mathrm{d} x^{\mu}$. Knowing that the action of a vector field on a form is given via Lie derivative one can show that

$$
\begin{aligned}
& X_{1}\left(\mathrm{~d} x^{\mu}\right)=0, \quad X_{2}\left(\mathrm{~d} x^{\mu}\right)=\delta_{j}^{\mu} \mathrm{d} x^{j}, \\
& \mathrm{~d} x^{\mu} \wedge \star \mathrm{d} x^{\nu}=\mathrm{d} x^{\mu} \wedge \mathrm{d} x^{\nu}=-\mathrm{d} x^{\nu} \wedge \mathrm{d} x^{\mu}=-\mathrm{d} x^{\nu} \wedge_{\star} \mathrm{d} x^{\mu}, \\
& f \star \mathrm{d} x^{0}=\mathrm{d} x^{0} \star f, \quad f \star \mathrm{d} x^{j}=\mathrm{d} x^{j} \star e^{i a \partial_{0}} f .
\end{aligned}
$$

Since basis 1-forms anticommute the volume form remains undeformed

$$
\mathrm{d}_{\star}^{4} x:=\mathrm{d} x^{0} \wedge_{\star} \mathrm{d} x^{1} \wedge_{\star} \cdots \wedge_{\star} \mathrm{d} x^{3}=\mathrm{d} x^{0} \wedge \mathrm{d} x^{1} \wedge \cdots \wedge \mathrm{d} x^{3}=\mathrm{d}^{4} x .
$$

The $\star$-derivatives follow from (2.8) and are given by

$$
\begin{aligned}
& \partial_{0}^{\star}=\partial_{0}, \quad \partial_{j}^{\star}=e^{-\frac{i}{2} a \partial_{0}} \partial_{j}, \\
& \partial_{0}^{\star}(f \star g)=\left(\partial_{0}^{\star} f\right) \star g+f \star\left(\partial_{0}^{\star} g\right), \quad \partial_{j}^{\star}(f \star g)=\left(\partial_{j}^{\star} f\right) \star e^{-i a \partial_{0}} g+f \star\left(\partial_{j}^{\star} g\right) .
\end{aligned}
$$

Arbitrary forms $\omega_{1}$ and $\omega_{2}$ do not anticommute, the $\wedge$ product is deformed to the $\star$-wedge product

$$
\omega_{1} \wedge_{\star} \omega_{2} \neq(-1)^{d_{1} \cdot d_{2}} \omega_{2} \wedge_{\star} \omega_{1},
$$

where $d_{1}$ and $d_{2}$ are the degrees of forms. However, under the integral forms anticommute

$$
\int \omega_{1} \wedge_{\star} \omega_{2}=(-1)^{d_{1} \cdot d_{2}} \int \omega_{2} \wedge_{\star} \omega_{1}, \quad \text { with } \quad d_{1}+d_{2}=4
$$


This holds because the twist (4.2) fulfils the property $S\left(\bar{f}^{\alpha}\right) \bar{f}_{\alpha}=1$. The property (4.7) can be generalized to

$$
\int \omega_{1} \wedge_{\star} \cdots \wedge_{\star} \omega_{p}=(-1)^{d_{1} \cdot d_{2} \cdots \cdot d_{p}} \int \omega_{p} \wedge_{\star} \omega_{1} \wedge_{\star} \cdots \wedge_{\star} \omega_{p-1}, \text { with } d_{1}+d_{2}+\cdots+d_{p}=4
$$

We say that the integral is cyclic. This property is very important for construction of NC gauge theories.

\section{$4.2 U(1)$ gauge theory}

In order to construct the $\mathrm{NC} U(1)$ gauge theory we now use the SW map solutions from Section 2. We expand the recursive relations up to the first order in the NC parameter $a$ and use the particular form of the twist (4.2). Also, when writing the expanded solutions for the components of forms we have to use (4.5).

Expanding (3.4) we obtain the components of the gauge field $\hat{A}=\hat{A}_{\mu} \star \mathrm{d} x^{\mu}$ :

$$
\hat{A}_{\mu}=A_{\mu}-\frac{a}{2} \delta_{\mu}^{j}\left(i \partial_{0} A_{j}+A_{0} A_{j}\right)+\frac{1}{2} C_{\lambda}^{\rho \sigma} x^{\lambda}\left(F_{\rho \mu} A_{\sigma}-A_{\rho} \partial_{\sigma} A_{\mu}\right)
$$

with the commutative gauge field $A_{\mu}$ The first order solution of the field-strength tensor $\hat{F}=$ $\frac{1}{2} \hat{F}_{\mu \nu} \star \mathrm{d} x^{\mu} \wedge_{\star} \mathrm{d} x^{\nu}$ follows from (3.5) and is given by

$$
\begin{aligned}
& \hat{F}_{0 j}=F_{0 j}-\frac{i a}{2} \partial_{0} F_{0 j}-a A_{0} F_{0 j}+C_{\lambda}^{\rho \sigma} x^{\lambda}\left(F_{\rho 0} F_{\sigma j}-A_{\rho} \partial_{\sigma} F_{0 j}\right), \\
& \hat{F}_{i j}=F_{i j}-i a \partial_{0} F_{i j}-2 a A_{0} F_{i j}+C_{\lambda}^{\rho \sigma} x^{\lambda}\left(F_{\rho i} F_{\sigma j}-A_{\rho} \partial_{\sigma} F_{i j}\right) .
\end{aligned}
$$

Finally, in order to write a NC $U(1)$ gauge invariant action we need a NC Hodge dual of the field-strength tensor $\hat{F}$. We label it with $* \hat{F}$; it should have the following properties:

$$
\begin{aligned}
& \delta_{\alpha}^{\star}(* \hat{F})=i\left[\hat{\Lambda}_{\alpha}, * \hat{F}\right], \\
& \lim _{a \rightarrow 0}(* \hat{F})_{\mu \nu}=\frac{1}{2} \epsilon_{\mu \nu \rho \sigma} F^{\rho \sigma},
\end{aligned}
$$

with $F^{\rho \sigma}=\eta^{\rho \mu} \eta^{\sigma \nu} F_{\mu \nu}$. We use the flat metric $\eta_{\mu \nu}$ to raise and lower indices. The natural guess for the components of the NC Hodge dual

$$
(* \hat{F})_{\mu \nu}=\frac{1}{2} \epsilon_{\mu \nu \alpha \beta} \hat{F}^{\alpha \beta},
$$

gives a 2 -form $* \hat{F}=\frac{1}{2}(* \hat{F})_{\mu \nu} \star \mathrm{d} x^{\mu} \wedge_{\star} \mathrm{d} x^{\nu}$ which does not fulfil the property (4.10). Therefore, the action constructed using (4.12)

$$
S=\frac{1}{2} \int \hat{F} \wedge_{\star} * \hat{F}
$$

is not gauge invariant. The construction of the Hodge dual on NC spaces turns out to be a problem in general. In some simple examples, like $\theta$-constant deformation, the natural guess (4.12) works well, but for other more complicated deformations this is not the case. There are different ways of solving (or at least going around) this problem and we describe some of them next. 


\subsection{Discussion}

In the following, we discuss three different ways of overcoming the problem of defining the NC Hodge dual.

Method 1. We introduce a two form $\hat{Z}=\frac{1}{2}(\hat{Z})_{\mu \nu} \star \mathrm{d} x^{\mu} \wedge_{\star} \mathrm{d} x^{\nu}$ as

$$
\hat{Z}=\frac{1}{2} \epsilon_{\mu \nu \alpha \beta} \hat{Z}^{\alpha \beta} \star \mathrm{d} x^{\mu} \wedge_{\star} \mathrm{d} x^{\nu}
$$

and demand that it fulfils the properties (4.10) and (4.11). Note that $\hat{Z}_{\mu \nu}=\epsilon_{\mu \nu \alpha \beta} \hat{Z}^{\alpha \beta}$. Then, using the first property, we solve the SW map for this two form. Since it transforms in the adjoint representation, (4.10), the recursive relation is given by

$$
\hat{Z}^{(n+1)}=-\frac{1}{4(n+1)} \theta^{C D}\left(\left\{\hat{A}_{C} \stackrel{\star}{,}\left(l_{D}+L_{D}\right) \hat{Z}\right\}\right)^{(n)} .
$$

Expanding this relation up to first order in the NC parameter $a$ and using the twist (4.2) we obtain

$$
\begin{aligned}
& \hat{Z}^{0 j}=F^{0 j}-i a \partial_{0} F^{0 j}-2 a A_{0} F^{0 j}+C_{\lambda}^{\rho \sigma} x^{\lambda}\left(F_{\rho}^{0} F_{\sigma}^{j}-A_{\rho} \partial_{\sigma} F^{0 j}\right), \\
& \hat{Z}^{i j}=F^{i j}-\frac{i a}{2} \partial_{0} F^{i j}-a A_{0} F^{i j}+C_{\lambda}^{\rho \sigma} x^{\lambda}\left(F_{\rho}^{i} F_{\sigma}^{j}-A_{\rho} \partial_{\sigma} F^{i j}\right) .
\end{aligned}
$$

The NC gauge invariant action can be written as

$$
S_{1}=\frac{1}{2} \int \hat{F} \wedge_{\star} \hat{Z}=-\frac{1}{4} \int\left\{2 \hat{F}_{0 j} \star e^{-i a \partial_{0}} \hat{Z}^{0 j}+\hat{F}_{i j} \star e^{-2 i a \partial_{0}} \hat{Z}^{i j}\right\} \star \mathrm{d}^{4} x .
$$

The terms $e^{-i a \partial_{0}} \hat{Z}^{0 j}$ and $e^{-2 i a \partial_{0}} \hat{Z}^{i j}$ come from $\star$-commuting basis 1 -forms with the components $\hat{Z}^{\mu \nu}$. Inserting the SW map solutions (4.8), (4.9), (4.13) and (4.14) into (4.15) leads to

$$
S_{1}=-\frac{1}{4} \int \mathrm{d}^{4} x\left\{F_{\mu \nu} F^{\mu \nu}-\frac{1}{2} C_{\lambda}^{\rho \sigma} x^{\lambda} F^{\mu \nu} F_{\mu \nu} F_{\rho \sigma}+2 C_{\lambda}^{\rho \sigma} x^{\lambda} F^{\mu \nu} F_{\mu \rho} F_{\nu \sigma}\right\} .
$$

This action is invariant under the commutative $U(1)$; this is guaranteed by the SW map. One can further study coupling to the matter fields, equations of motion and their solutions. It seems that this method works fine. On the other hand, we had to introduce an additional field $\hat{Z}$ as a replacement for the NC Hodge dual of the field-strength tensor. This field is an independent field, not a function of $\hat{F}$ as in the case of the Hodge dual. After the expansion in the commutative fields, we see that there are no new degrees of freedom; SW map takes care of that. However, if one discusses the freedom of the SW map [43] one finds additional covariant terms that enter the action (4.16) with arbitrary coefficients. These terms could be fixed by imposing some additional physical requirements.

Method 2. If we look closely at the problem of definition of the Hodge dual, we see that the problem arises because the basis 1 -forms do not $\star$-commute with functions (4.6). But we are free to choose a different basis, because we write the action in a basis independent form. Instead of working in the coordinate basis, we now redo calculations in the natural basis (frame in the sense of Madore [40]). This basis is defined as a basis in which basis 1-forms $\theta^{a} \star$-commute with functions, $f \star \theta^{a}=\theta^{a} \star f$. Its particular form will in general depend on the choice of the twist. For the twist (4.2) it is given by [48]

$$
\begin{aligned}
& x^{\mu}=\left(t=x^{0}, x, y, z\right), \quad \mathrm{d} x^{\mu}=(\mathrm{d} t, \mathrm{~d} x, \mathrm{~d} y, \mathrm{~d} z), \quad \partial_{\mu}=\left(\partial_{t}, \partial_{x}, \partial_{y}, \partial_{z}\right) \\
& \downarrow \\
& x^{a}=(t, r, \theta, \varphi), \quad \theta^{a}=\left(\mathrm{d} t, \frac{\mathrm{d} r}{r}, \mathrm{~d} \theta, \mathrm{d} \varphi\right), \quad e_{a}=\left(\partial_{t}, r \partial_{r}, \partial_{\theta}, \partial_{\varphi}\right) .
\end{aligned}
$$


The twist (4.2) is then rewritten as

$$
\mathcal{F}=e^{-\frac{i}{2} \theta^{a b} X_{a} \otimes X_{b}}=e^{-\frac{i a}{2}\left(\partial_{t} \otimes r \partial_{r}-r \partial_{r} \otimes \partial_{t}\right)}
$$

with $X_{1}=\partial_{t}=e_{0}$ and $X_{2}=r \partial_{r}=e_{1}$. The $\star$-product in this basis is

$$
\begin{aligned}
f \star g & =f \cdot g+\frac{i a}{2}\left(\left(e_{0} f\right)\left(e_{1} g\right)-\left(e_{1} f\right)\left(e_{0} g\right)\right)+\mathcal{O}\left(a^{2}\right) \\
& =f \cdot g+\frac{i a}{2}\left(\left(\partial_{t} f\right)\left(r \partial_{r} g\right)-\left(r \partial_{r} f\right)\left(\partial_{t} g\right)\right)+\mathcal{O}\left(a^{2}\right) .
\end{aligned}
$$

Note that the new basis is not flat, the metric is given by $g_{a b}=\operatorname{diag}\left(1,-r^{2},-r^{2},-r^{2} \sin ^{2} \theta\right)$. Since the metric does not depend on the coordinate $t$ the $\star$-inverse is the same as the usual inverse $g_{a b} \star g^{a c}=g_{a b} g^{a c}=\delta_{a}^{c}$. The volume element is

$$
d^{4} x=\sqrt{-g} \epsilon_{a b c d} \theta^{a} \wedge \theta^{b} \wedge \theta^{c} \wedge \theta^{d}=r^{2} \sin \theta \mathrm{d} t \mathrm{~d} r \mathrm{~d} \theta \mathrm{d} \varphi .
$$

The Hodge dual $* \hat{F}$ we define generalizing the usual expression for the Hodge dual in curved space given by

$$
* F^{(0)}=\frac{1}{2} \epsilon_{a b c d} \sqrt{-g} g^{a e} g^{b f} F_{e f}^{(0)} \theta^{c} \wedge \theta^{d} .
$$

In order to have a $\mathrm{NC}$ gauge invariant action it is necessary that $* \hat{F}$ transforms covariantly under NC gauge transformations. To ensure this we have to covariantize the expression $\sqrt{-g} g^{a e} g^{b f}$. We define

$$
* \hat{F}=\frac{1}{2} \epsilon_{a b c d} \hat{G}^{a e b f} \star \hat{F}_{e f} \star \theta^{c} \wedge_{\star} \theta^{d} .
$$

Here $\hat{G}^{a e b f}$ is the quantity that under NC gauge transformations transforms covariantly

$$
\delta_{\alpha}^{\star} \hat{G}^{a e b f}=i\left[\hat{\Lambda}_{\alpha}^{\star}, \hat{G}^{a e b f}\right] .
$$

and in the limit $a \rightarrow 0$ reduces to $\sqrt{-g} g^{a e} g^{b f}$. The SW map solution for $\hat{G}^{a e b f}$ up to first order in $a$ is given by

$$
\hat{G}^{a e b f}=\sqrt{-g} g^{a e} g^{b f}-a A_{0} e_{1}\left(\sqrt{-g} g^{a e} g^{b f}\right) .
$$

The SW map solutions for $A$ and $F$ in the new basis are

$$
\begin{aligned}
& \hat{A}_{a}=A_{a}^{0}+\frac{a}{2}\left(A_{1}^{0} F_{0 a}^{0}-A_{0}^{0} F_{1 a}^{0}+A_{1}^{0}\left(e_{0} A_{a}\right)-A_{0}^{0}\left(e_{1} A_{a}\right)\right), \\
& \hat{F}_{a b}=F_{a b}^{0}+a\left(F_{0 a}^{0} F_{1 b}^{0}-F_{1 a}^{0} F_{0 b}^{0}-A_{0}^{0}\left(e_{1} F_{a b}^{0}\right)+A_{1}^{0}\left(e_{0} F_{a b}^{0}\right)\right) .
\end{aligned}
$$

Finally, we construct and expand the $\mathrm{NC} U(1)$ gauge invariant action and obtain

$$
S_{2}=\frac{1}{2} \int(* \hat{F}) \wedge_{\star} \hat{F}=-\frac{1}{4} \int \mathrm{d}^{4} x\left\{F_{a b}^{0} F^{0 a b}+a F^{0 a b}\left(4 F_{0 a}^{0} F_{1 b}^{0}-F_{01}^{0} F_{a b}^{0}\right)\right\},
$$

with $\mathrm{d}^{4} x=r^{2} \sin \theta \mathrm{d} t \mathrm{~d} r \mathrm{~d} \theta \mathrm{d} \varphi$. Note that the action (4.17) has the same form as the expanded action for the $\mathrm{NC}$ gauge field in the case of $\theta$-constant deformation [31, 30]. This is the consequence of the particular choice of basis, the natural basis, in which the twist looks like the Moyal-Weyl twist. One can do a coordinate transformation and write back the action (4.17) in the coordinate basis. The result (as expected) is (4.16). Concerning the degrees of freedom, the situation is the same as in Method 1 . There we introduced the field $\hat{Z}$, while in Method 2 
we introduced the field $\hat{G}^{a e b f}$. Therefore, the SW map freedom will contribute here as well and (we expect) with the same number of terms, since both $\hat{Z}$ and $\hat{G}$ transform covariantly.

Method 3. In [4] yet another approach is discussed. The action for the commutative $U(1)$ gauge theory coupled to gravity can be defined as ${ }^{6}$

$$
S=\frac{1}{2} \int \epsilon_{\mu \nu \rho \sigma}\left(f^{\mu \nu} F-\frac{1}{12} f_{\alpha \beta} f^{\alpha \beta} V^{\mu} \wedge V^{\nu}\right) \wedge V^{\rho} \wedge V^{\sigma} .
$$

Three fields appear in this action: the 2-form field-strength tensor $F=\frac{1}{2} F_{\mu \nu} \mathrm{d} x^{\mu} \wedge \mathrm{d} x^{\nu}$, the 1-form vierbein $V^{\mu}=\mathrm{d} x^{\mu}$ and the auxiliary field $f_{\alpha \beta}$. We work in the flat space-time: indices $\mu, \nu, \ldots$ are flat indices ${ }^{7}$ and are raised and lowered with the flat metric $\eta_{\mu \nu}$. Under the commutative $U(1)$ gauge transformation all three fields are invariant and therefore the action (4.18) is also invariant. The equation of motion for the field $f_{\alpha \beta}$ identifies $f_{\alpha \beta}=\frac{1}{2} F_{\alpha \beta}$. Inserting this into the action (4.18) gives

$$
S=-\frac{1}{4} \int \mathrm{d}^{4} x F_{\mu \nu} F^{\mu \nu}
$$

We see that by introducing the auxiliary field $f_{\alpha \beta}$ one can avoid the explicit use of the Hodge dual in the action. All this should now be generalized to the NC spaces.

We define the NC $U(1)$ gauge field action as

$$
S_{3}=\frac{1}{2} \int \epsilon_{\mu \nu \rho \sigma}\left(\frac{1}{2}\left(\hat{f}^{\mu \nu} \star \hat{F}+\hat{F} \star \hat{f}^{\mu \nu}\right)-\frac{1}{12} \hat{f}_{\alpha \beta} \star \hat{f}^{\alpha \beta} \star \hat{V}^{\mu} \wedge_{\star} \hat{V}^{\nu}\right) \wedge_{\star} \hat{V}^{\rho} \wedge_{\star} \hat{V}^{\sigma}
$$

with noncommutative fields $\hat{f}_{\alpha \beta}, \hat{F}$ and $\hat{V}^{\mu}$. Note that in order to have a hermitean action we had to symmetrize the first term. All these fields transform covariantly, that is

$$
\delta_{\alpha}^{\star} \hat{F}=i\left[\hat{\Lambda}_{\alpha}^{\star} \stackrel{\hat{F}}{ }\right], \quad \delta_{\alpha}^{\star} \hat{f}_{\alpha \beta}=i\left[\hat{\Lambda}_{\alpha}^{\star \star}, \hat{f}_{\alpha \beta}\right], \quad \delta_{\alpha}^{\star} \hat{V}^{\mu}=i\left[\hat{\Lambda}_{\alpha} \stackrel{\star}{,} \hat{V}^{\mu}\right] .
$$

The next step is to solve the SW map for these fields and expand the action. Calculating the equations of motion and inserting them into the expanded action results in the on-shell action.

Solution for the field-strength tensor we already have; it is given by (3.5). The solutions for other fields are easy to find since these fields transform in the adjoint representation of the NC $U(1)$. The solutions are given by

$$
\begin{aligned}
& \hat{f}_{\alpha \beta}^{(n+1)}=-\frac{1}{4(n+1)} \theta^{C D}\left(\left\{\hat{A}_{C}{ }^{\star},\left(l_{D}+L_{D}\right) \hat{f}_{\alpha \beta}\right\}\right)^{(n)}, \\
& \hat{V}^{\mu(n+1)}=-\frac{1}{4(n+1)} \theta^{C D}\left(\left\{\hat{A}_{C}^{\star},\left(l_{D}+L_{D}\right) \hat{V}^{\mu}\right\}\right)^{(n)} .
\end{aligned}
$$

Inserting these solutions in the action (4.19) and expanding up to first order in the deformation parameter $a$ we obtain

$$
\begin{aligned}
S_{3}= & \frac{1}{2} \int \epsilon_{\mu \nu \rho \sigma}\left(f^{\mu \nu} F-\frac{1}{12} f_{\alpha \beta} f^{\alpha \beta} V^{\mu} \wedge V^{\nu}\right) \wedge V^{\rho} \wedge V^{\sigma} \\
& +\frac{1}{4} \theta^{C D} \int \epsilon_{\mu \nu \rho \sigma}\left(F_{C D} f^{\mu \nu} F \wedge V^{\rho} \wedge V^{\sigma}+f^{\mu \nu} F_{C} \wedge F_{D} \wedge V^{\rho} \wedge V^{\sigma}\right. \\
& \left.-\frac{1}{12} F_{C D} f_{\alpha \beta} f^{\alpha \beta} V^{\mu} \wedge V^{\nu} \wedge V^{\rho} \wedge V^{\sigma}\right) .
\end{aligned}
$$

\footnotetext{
${ }^{6}$ In the paper [4] the authors consider a more general case: non-Abelian gauge theory coupled to gravity. Here we concentrate only on the $U(1)$ theory in flat space-time.

${ }^{7}$ The standard notation in gravity is that greek indices are curved (Einstein) indices while Latin indices are flat (Lorentz) indices. However, in our case the greek indices are flat because we work in the flat space-time. That is, we apply the method of [4] but in a very simple case: flat space-time and $U(1)$ gauge theory.
} 
The one-form $F_{C}$ is obtained by contraction along the vector field $X_{C}, F_{C}=i_{X_{C}} F$ and the zeroform $F_{C D}$ is defined as a double contraction $F_{C D}=i_{X_{C}} i_{X_{D}} F$ and can be rewritten in terms of Lie derivatives of the connection one-form $A$ as $F_{C D}=-l_{C} A_{D}+l_{D} A_{C}$ with $A_{C}=i_{X_{C}} A$. The vector fields $X_{C}, X_{D}$ are defined in (4.2). More explicitly, the expanded action (4.20) is given by

$$
\begin{aligned}
S_{3}= & \int \mathrm{d}^{4} x\left(-F^{\alpha \beta} f_{\alpha \beta}+f^{\alpha \beta} f_{\alpha \beta}-\frac{1}{2} C_{\lambda}^{\rho \sigma} x^{\lambda} F_{\rho \sigma}\left(-F^{\alpha \beta} f_{\alpha \beta}+f^{\alpha \beta} f_{\alpha \beta}\right)\right. \\
& \left.-C_{\lambda}^{\rho \sigma} x^{\lambda} f^{\alpha \beta} F_{\rho \alpha} F_{\sigma \beta}\right) .
\end{aligned}
$$

Varying this action with respect to $f_{\alpha \beta}$ gives the equation of motion for this field

$$
f_{\alpha \beta}\left(1-\frac{1}{2} C_{\lambda}^{\rho \sigma} x^{\lambda} F_{\rho \sigma}\right)=\frac{1}{2} F_{\alpha \beta}-\frac{1}{4} C_{\lambda}^{\rho \sigma} x^{\lambda} F_{\rho \sigma} F_{\alpha \beta}+\frac{1}{2} C_{\lambda}^{\rho \sigma} x^{\lambda} F_{\rho \alpha} F_{\sigma \beta},
$$

with the solution up to first order in the deformation parameter $a$

$$
f_{\alpha \beta}=\frac{1}{2} F_{\alpha \beta}+\frac{1}{2} C_{\lambda}^{\rho \sigma} x^{\lambda} F_{\rho \alpha} F_{\sigma \beta} .
$$

Inserting this solution into the action (4.21) gives to on-shell action

$$
S_{3}=-\frac{1}{4} \int \mathrm{d}^{4} x\left\{F_{\mu \nu} F^{\mu \nu}-\frac{1}{2} C_{\lambda}^{\rho \sigma} x^{\lambda} F^{\mu \nu} F_{\mu \nu} F_{\rho \sigma}+2 C_{\lambda}^{\rho \sigma} x^{\lambda} F^{\mu \nu} F_{\mu \rho} F_{\nu \sigma}\right\} .
$$

This is exactly the result we obtained in Method 1 (4.16) and Method 2 (4.17).

Finally, to conclude this analysis: There are different ways to solve (or at least to go around) the problem of the definition of NC Hodge dual. What seems to be common for all of them is the introduction of an additional NC field in the adjoint representation. These fields do not change the number of degrees of freedom due to the SW map, but they can introduce additional covariant terms in the expanded actions provided one discusses the freedom of the SW map. Then these additional terms can be used to render some nice properties of the theory, like renormalizability [12].

\section{Kappa-Minkowski from a Jordanian twist}

The twisted symmetry of the $\kappa$-Minkowski space-time constructed in Section 4 is the twisted igl $(1,3)$. However, we would like to stay as close as possible to the Poincaré symmetry, that is we do not want to enlarge the symmetry algebra too much. Therefore, in this section we discuss twisting of the Poincaré-Weyl algebra denoted by iwso(1,3). This algebra has 11 generators: 10 of the Poincaré algebra and the dilatation generator $J$. The algebra is given by (4.1) and additional commutators:

$$
\left[M_{\mu \nu}, J\right]=0, \quad\left[J, P_{\mu}\right]=P_{\mu} .
$$

In the natural representation the (anti-hermitean) generators are given by $M_{\mu \nu}=x_{\mu} \partial_{\nu}-x_{\nu} \partial_{\mu}$, $P_{\mu}=\partial_{\mu}$ and $J=-x^{\mu} \partial_{\mu}$. The universal enveloping algebra of this algebra is $\operatorname{Uiwso}(1,3)$; it becomes a Hopf algebra with the structure (2.1).

We use the so-called Jordanian twist to deform Uiwso(1,3). Generally Jordanian twists are related with the Borel subalgebra of a given Lie algebra: $\mathfrak{b}^{2}=\{h, e \mid[h, e]=e\} .{ }^{8}$ Such twists have the following form $[24,45]$

$$
\mathcal{F}_{\text {Jor }}=\exp (h \otimes \sigma),
$$

\footnotetext{
${ }^{8} \mathfrak{b}^{2}$ is isomorphic to the 2-dimensional solvable Lie algebra $\mathfrak{a n}^{1}$.
} 
where $\sigma=\ln (1+\lambda e)$ with the deformation parameter $\lambda$. These kind of twists can be symmetrized as shown in $[25,46,52]$.

In order to have a hermitean $\star$-product we work with the symmetrized version of Jordanian twist related with the Borel subalgebra of $i w s o(1,3)$ given by dilatation $J$ and momenta $P_{0}$ generators: $\left[J, P_{0}\right]=P_{0}$. The inverse of such symmetrized Jordanian twist is given by $[25]$ :

$$
\mathcal{F}^{-1}=\sum_{m=0}^{\infty} \frac{1}{m !}\left(-\frac{i a}{2}\right)^{m} \sum_{r=0}^{m}(-1)^{r}\left(\begin{array}{c}
m \\
r
\end{array}\right) P_{0}^{m-r} J^{\langle r\rangle} \otimes P_{0}^{r} J^{\langle m-r\rangle},
$$

where the following notation is used:

$$
J^{\langle 0\rangle}=1, \quad J^{\langle r\rangle}=J(J+1) \cdots(J+r-1), \quad r=1,2, \ldots
$$

Under the action of the twist the algebra relations do not change. However, the coalgebra sector is deformed. We give here the deformed coproduct for momenta generators only

$$
\Delta^{\mathcal{F}}\left(P_{\mu}\right)=\sum_{m=0}^{\infty}\left[(-1)^{m}\left(\frac{i a}{2}\right)^{2 m}\left(P_{0} \otimes P_{0}\right)^{m}\left[\Delta_{0}\left(P_{\mu}\right)+\frac{i a}{2}\left(P_{0} \otimes P_{\mu}-P_{\mu} \otimes P_{0}\right)\right]\right] .
$$

It will be used to calculate the coproduct for the new derivatives $\partial_{\mu}^{\star}$ in the next subsection. For the rest of deformed coproducts we refer the reader to the Appendix. Once again, the twisted Hopf algebra is not the $\kappa$-Poincaré algebra from $[38,37]$. It is the twisted $U_{\text {iwso(1,3) }}^{\mathcal{F}}[[a]]$.

The inverse of the twist defines the $\star$-product between functions and in a compact form can be written in the following way:

$$
\begin{aligned}
f \star g & =\mu\left\{\mathcal{F}^{-1} f \otimes g\right\} \\
& =\mu\left[\sum_{m=0}^{\infty} \frac{1}{m !}\left(-\frac{i a}{2}\right)^{m} \sum_{r=0}^{m}(-1)^{r}\left(\begin{array}{c}
m \\
r
\end{array}\right) P_{0}^{m-r} J^{\langle r\rangle}(f) \otimes P_{0}^{r} J^{\langle m-r\rangle}(g)\right] .
\end{aligned}
$$

For the future use we rewrite $\mathcal{F}^{-1}$ order by order, using expansion in the deformation parameter $a$ :

$$
\begin{aligned}
\mathcal{F}^{-1}= & 1 \otimes 1-\frac{i a}{2}\left(P_{0} \otimes J-J \otimes P_{0}\right) \\
& +\frac{(i a)^{2}}{8}\left(P_{0}^{2} \otimes J(J+1)-2 P_{0} J \otimes P_{0} J+J(J+1) \otimes P_{0}^{2}\right) \\
& -\frac{(i a)^{3}}{8} \frac{1}{3 !}\left[P_{0}^{3} \otimes J(J+1)(J+2)\right. \\
& \left.-3 P_{0}^{2} J \otimes P_{0} J(J+1)+3 P_{0} J(J+1) \otimes P_{0}^{2} J-J(J+1)(J+2) \otimes P_{0}^{3}\right]+\mathcal{O}\left(a^{4}\right) .
\end{aligned}
$$

The $\star$-product is then

$$
\begin{aligned}
f \star g= & \mu\left\{\mathcal{F}^{-1} f \otimes g\right\}=f \cdot g+i \frac{a}{2} x^{j}\left(\partial_{0} f \partial_{j} g-\partial_{j} f \partial_{0} g\right) \\
& +\frac{a^{2}}{8}\left(-x^{i} x^{j}\left[\partial_{0}^{2} f\left(\partial_{i} \partial_{j} g\right)+\left(\partial_{i} \partial_{j} f\right) \partial_{0}^{2} g-2\left(\partial_{0} \partial_{i} f\right)\left(\partial_{0} \partial_{j} g\right)\right]\right. \\
& \left.+2 \partial_{0} f \partial_{0} g+2 x^{\rho} \partial_{0} f \partial_{0} \partial_{\rho} g+2 x^{\mu} \partial_{0} \partial_{\mu} f \partial_{0} g\right)+\mathcal{O}\left(a^{3}\right) .
\end{aligned}
$$

The commutation relations between coordinates are the $\kappa$-Minkowski commutation relations:

$$
\left[x^{0}, x^{j}\right]=x^{0} \star x^{j}-x^{j} \star x^{0}=i a x^{j}, \quad\left[x^{i} \star x^{j}\right]=0 .
$$

Note that the star product for $\kappa$-Minkowski space-time up to the first order is the same when coming from the Jordanian twist (also the non-symmetric one, see, e.g., [10]) and from the Abelian twist (4.4). 


\subsection{Twisted differential calculus}

The usual exterior derivative is the $\star$-exterior derivative. As in Section 4, we use the coordinate basis; the basis 1-forms are $\mathrm{d} x^{\mu}$. As the action of a vector field on a form is given via Lie derivative, we obtain

$$
P_{0}\left(\mathrm{~d} x^{\mu}\right)=0, \quad J\left(\mathrm{~d} x^{\mu}\right)=-\mathrm{d} x^{\mu} .
$$

Using these relations one can show that the basis 1-forms anticommute

$$
\mathrm{d} x^{\mu} \wedge_{\star} \mathrm{d} x^{\nu}=\mathrm{d} x^{\mu} \wedge \mathrm{d} x^{\nu}=-\mathrm{d} x^{\nu} \wedge \mathrm{d} x^{\mu}=-\mathrm{d} x^{\nu} \wedge_{\star} \mathrm{d} x^{\mu},
$$

but do not $\star$-commute with functions

$$
f \star \mathrm{d} x^{\mu}=f \mathrm{~d} x^{\mu}+\frac{i a}{2} \partial_{0} f \mathrm{~d} x^{\mu}, \quad \mathrm{d} x^{\mu} \star f=f \mathrm{~d} x^{\mu}-\frac{i a}{2} \partial_{0} f \mathrm{~d} x^{\mu} .
$$

Note that the relations (5.3) are valid to all orders in $a$.

One can rewrite the usual exterior derivative of a function using the $\star$-product as

$$
\mathrm{d} f=\left(\partial_{\mu} f\right) \mathrm{d} x^{\mu}=\left(\partial_{\mu}^{\star} f\right) \star \mathrm{d} x^{\mu},
$$

where the new derivatives $\partial_{\mu}^{\star}$ are defined by this equation. We obtain the following relation, again valid to all orders in $a$ :

$$
\left(1+\frac{i a}{2} \partial_{0}\right) \partial_{\mu}^{\star}=\partial_{\mu}
$$

However, the coproduct for the new derivatives $\partial_{\mu}^{\star}$ can only be calculated order by order, using (5.2) and the expansion in $a$. Up to second order we obtain:

$$
\Delta\left(\partial_{\mu}^{\star}\right)=\partial_{\mu}^{\star} \otimes 1+1 \otimes \partial_{\mu}^{\star}-i a \partial_{\mu}^{\star} \otimes \partial_{0}^{\star}+\mathcal{O}\left(a^{3}\right) .
$$

Unfortunately, the twist (5.1) does not fulfil $S\left(\bar{f}^{\alpha}\right) \bar{f}_{\alpha}=1$ and the integral will not be cyclic. This is a problem when one wants to discuss NC gauge theories and use the variational principle. We postpone the discussion until next subsection.

\section{$5.2 U(1)$ gauge theory}

Having defined differential calculus we are ready to formulate NC gauge theory on $\kappa$-Minkowski space-time possessing the twisted Weyl-Poincaré symmetry. We use the SW map solutions from Section 3 and expand everything up to the first order in the deformation parameter $a$. Strictly speaking, formulae from the Section 3 are only valid in the case of Abelian twist deformation and will not be valid in the case of Jordanian deformation (5.1). But since the first order of the *-product is the same for both twists we can use these solutions up to first order ${ }^{9}$.

Let us write the first order expansions of the SW map solutions. The NC gauge parameter $\hat{\Lambda}_{\alpha}$ up to first order in the NC parameter $a$ is given by

$$
\hat{\Lambda}_{\alpha}=\alpha-\frac{1}{2} C_{\lambda}^{\mu \nu} x^{\lambda} A_{\mu}^{0} \partial_{\nu} \alpha
$$

\footnotetext{
${ }^{9}$ One can also solve the SW map equations from a scratch, order by order in the case of Jordanian twist. We did that and found that the first order solutions coincide with the first order expansion of solutions in Section 3. However, higher order solutions will be different for the Abelian and the Jordanian deformation.
} 
The NC gauge field $\hat{A}=\hat{A}_{\mu} \star \mathrm{d} x^{\mu}$ we calculate from (3.4)

$$
\hat{A}_{\mu}=A_{\mu}-\frac{a}{2}\left(i \partial_{0} A_{\mu}+A_{0} A_{\mu}\right)-\frac{1}{2} C_{\lambda}^{\rho \sigma} x^{\lambda} A_{\rho}\left(\partial_{\sigma} A_{\mu}^{0}+F_{\sigma \mu}\right) .
$$

The components of the field-strength tensor $\hat{F}=\frac{1}{2} \hat{F}_{\mu \nu} \star \mathrm{d} x^{\mu} \wedge_{\star} \mathrm{d} x^{\nu}$ follow from (3.5)

$$
\hat{F}_{\mu \nu}=F_{\mu \nu}-i a \partial_{0} F_{\mu \nu}-2 a A_{0} F_{\mu \nu}-C_{\lambda}^{\rho \sigma} x^{\lambda}\left(A_{\rho} \partial_{\sigma} F_{\mu \nu}-F_{\rho \mu} F_{\sigma \nu}\right) .
$$

Note that this solution is different then (4.8) and (4.9). This is a consequence of the Jordanian deformation and the difference in the differential calculus, compare (4.6) and (5.3). Next step is the construction of gauge invariant action. Here we face the following problems:

1. In order to write a $\mathrm{NC}$ action for the gauge fields, we need a $\mathrm{NC}$ generalization of the Hodge dual of the field-strength tensor $\hat{F}$. The problem is the same as in Section 4.

2. The integral is not cyclic.

In Section 4 we saw that there are three ways to "step around" the problem 1 . In the case of Jordanian deformation, due to the non-cyclicity of the integral, problems 1 and 2 interfere and cannot be analyzed separately. To solve this we will use a modification of the first method in Section 4. The other methods we comment in the next subsection.

We modify the integral by introducing a measure function $\mu(x)$ in the following way

$$
\int \mu(x) \cdot\left(\omega_{1} \wedge_{\star} \omega_{2}\right)=\int \mu(x) \cdot\left(\omega_{1} \wedge \omega_{2}\right) .
$$

Of course, to define an integral $\omega_{1} \wedge_{\star} \omega_{2}$ has to be a maximal form. Now one has to perform the explicit calculation, expanding the $\star$-product and taking into the account that vector fields from the definition of the twist act on forms via the Lie derivative and that Lie derivatives fulfil the Leibniz rule. We do the calculation up to the first order in the deformation parameter, but it can be generalized to higher orders. The first order of (5.5) is given by

$$
\begin{aligned}
& -\frac{i a}{2} \int \mu(x)\left(\left(l_{P_{0}} \omega_{1}\right) \wedge\left(l_{J} \omega_{2}\right)-\left(l_{J} \omega_{1}\right) \wedge\left(l_{P_{0}} \omega_{2}\right)\right) \\
& \quad=\frac{i a}{2} \int\left(\left(l_{P_{0}} \mu\right) \omega_{1} \wedge l_{J} \omega_{2}+\mu \omega_{1} \wedge l_{\left[P_{0}, J\right]} \omega_{2}-\left(l_{J} \mu\right) \omega_{1} \wedge\left(l_{P_{0}} \omega_{2}\right)\right) .
\end{aligned}
$$

Going from the first to the second line we performed integration by parts and discarded the surface terms. Knowing that $\left[J, P_{0}\right]=P_{0}$, we obtain the following conditions on the measure function $\mu: l_{P_{0}} \mu=0$ and $l_{J} \mu=-\mu$. We checked that no new conditions on $\mu(x)$ appear in the second order, and we conjecture that this holds to all orders.

We see that the measure function is $a$-independent and does not vanish in the limit $a \rightarrow 0$. One possible solution in four dimensions is given by

$$
f(x)=\sqrt{\left(x_{1}\right)^{2}+\left(x_{3}\right)^{2}+\left(x_{3}\right)^{2}} .
$$

A more precise mathematical description (and justification) of adding the measure function (changing the volume element) can be found in [23].

Next, we need a Hodge dual to write a gauge invariant action for the NC gauge field. In addition, since the measure function $\mu$ does not vanish in the commutative limit, we have to find a way to cancel it from the zeroth order of the equations of motion. Having these two requirements in mind, we construct the following action

$$
S=\frac{1}{2} \int \mu(x) \cdot\left(\hat{Y} \wedge_{\star} \hat{F}\right),
$$


where $\mu(x)$ is defined by (5.5) and (5.6) and $\hat{Y}$ is a 2 -form which satisfies

$$
\begin{aligned}
& \hat{Y}=\frac{1}{2} \hat{Y}_{\mu \nu} \star \mathrm{d} x^{\mu} \wedge_{\star} \mathrm{d} x^{\nu}, \\
& \delta_{\alpha}^{\star} \hat{Y}=i\left[\Lambda_{\alpha} \stackrel{\star}{,} \hat{Y}\right], \\
& \lim _{a \rightarrow 0} \hat{Y}_{\mu \nu}=\frac{1}{\mu(x)} \frac{1}{2} \epsilon_{\mu \nu \rho \sigma} F^{\rho \sigma} .
\end{aligned}
$$

The action (5.7) is gauge invariant and the good commutative limit is ensured by (5.10). Expanding the action (5.7) we obtain

$$
S=\frac{1}{2} \int \mu(x) \cdot \frac{1}{4}\left(\hat{Y}_{\mu \nu} \star\left(\left(1-2 i a \partial_{0}\right) \hat{F}_{\rho \sigma}\right) \star \epsilon^{\mu \nu \rho \sigma} \mathrm{d}^{4} x\right) .
$$

The SW map solution for $\hat{Y}$ can be found solving perturbatively (5.9) and up to first order in $a$ is given by

$$
\hat{Y}_{\mu \nu}=\frac{1}{2 \mu(x)} \epsilon_{\mu \nu \rho \sigma}\left(F^{\rho \sigma}-a\left(i \partial_{0} F^{\rho \sigma}+A_{0} F^{\rho \sigma}+C_{\lambda}^{\alpha \beta} x^{\lambda} A_{\alpha} \partial_{\beta} F^{\rho \sigma}\right)\right) .
$$

Inserting the SW map solutions (5.4) and (5.12) into the action (5.11) we obtain

$$
S=-\frac{1}{4} \int \mathrm{d}^{4} x\left(F_{\mu \nu} F^{\mu \nu}+C_{\lambda}^{\rho \sigma} x^{\lambda}\left(F^{\mu \nu} F_{\mu \rho} F_{\nu \sigma}-\frac{1}{2} F^{\mu \nu} F_{\mu \nu} F_{\rho \sigma}\right)\right) .
$$

It is obvious that the obtained action is invariant under the commutative $U(1)$ gauge transformations, that is guaranteed by the SW map. The result is different than the one obtained in Section 4. We obtained the same terms as in (4.16), however the relative coefficient between the second and the third term is different compared with (4.16). This difference can be attributed to the properties of Jordanian twist, i.e., to the different twisted symmetry than in the previous case.

We can write both actions, (4.16) and (5.13), as

$$
S=-\frac{1}{4} \int \mathrm{d}^{4} x\left(F_{\mu \nu} F^{\mu \nu}+C_{\lambda}^{\rho \sigma} x^{\lambda}\left(n F^{\mu \nu} F_{\mu \rho} F_{\nu \sigma}-\frac{1}{2} F^{\mu \nu} F_{\mu \nu} F_{\rho \sigma}\right)\right),
$$

where the parameter $n$ takes value $n=2$ in the case of the Abelian twist and $n=1$ in the case of the Jordanian twist. The corresponding equations of motions are:

$$
\begin{aligned}
\partial_{\mu} F^{\mu \nu}= & a \frac{(6 n-4)}{4} F^{\nu \mu} F_{0 \mu}-a \frac{(3-2 n)}{4} \delta_{0}^{\nu} F^{\alpha \beta} F_{\alpha \beta}-C_{\lambda}^{\rho \sigma} x^{\lambda} \frac{n}{2}\left(F_{\rho}^{\mu} \partial_{\mu} F_{\sigma}^{\nu}+F_{\mu \sigma} \partial_{\rho} F^{\mu \nu}\right) \\
& -\frac{1}{4} C_{\lambda}^{\rho \sigma} x^{\lambda}(n-2) F^{\mu \nu} \partial_{\mu} F_{\rho \sigma}-\frac{1}{4} C_{\lambda}^{\nu \sigma} x^{\lambda}\left(1-\frac{n}{2}\right) \partial_{\sigma}\left(F_{\alpha \beta} F^{\alpha \beta}\right) .
\end{aligned}
$$

We notice that the last two terms in (5.14) are non-zero only for $n=1$, i.e., only in the case of the Jordanian twists. Moreover, the second term on the right-hand side changes sign in going from one case to the other. We conclude (on the level of action and equations of motion) that different twists lead to different physics. Next step would be the analysis of possible solutions of these equations, and we hope to address this issue in future.

\subsection{Discussion}

Modifying the first method of Section 4, we managed to construct the gauge invariant action in the case of the Jordanian twist. However, the origin of the measure function $\mu$ is not very clear. We can speculate that in higher dimensions the twist (5.1) looks simpler and when doing the 
dimensional reduction to four dimensions the twist obtains its Jordanian form and the measure function appears in the integral. So far we were not able to find a proper higher dimensional theory that could give all this.

There were two other methods discussed in Section 4. Let us comment briefly how they do (not) apply here. It is obvious that they have to be modified due to the non-cyclicity of the integral.

One can find a natural basis in the case of Jordanian deformation and it is given by

$$
\begin{aligned}
& x^{\mu}=\left(t=x^{0}, x, y, z\right), \quad \mathrm{d} x^{\mu}=(\mathrm{d} t, \mathrm{~d} x, \mathrm{~d} y, \mathrm{~d} z), \quad \partial_{\mu}=\left(\partial_{t}, \partial_{x}, \partial_{y}, \partial_{z}\right) \\
& \downarrow \\
& x^{a}=(t, r, \theta, \varphi), \quad \theta^{a}=\left(\frac{\mathrm{d} t}{r}, \frac{\mathrm{d} r}{r}, \mathrm{~d} \theta, \mathrm{d} \varphi\right), \quad e_{a}=\left(r \partial_{t}, r \partial_{r}, \partial_{\theta}, \partial_{\varphi}\right) .
\end{aligned}
$$

The vector fields entering the twist (5.1) are rewritten as $X_{1}=\partial_{0}=\frac{1}{r} e_{0}$ and $X_{2}=-x^{\mu} \partial_{\mu}=$ $-\frac{t}{r} e_{0}-e_{1}$. Then one can check that

$$
X_{1}\left(\theta^{a}\right)=0, \quad X_{2}\left(\theta^{a}\right)=0 .
$$

The basis 1-forms $\theta^{a}$ are frame 1-forms, they $\star$-commute with functions:

$$
\theta^{a} \star f=f \star \theta^{a}=f \cdot \theta^{a} .
$$

The construction of the Hodge dual is done following the same steps as in Section 4. Only when writing the integral, one has to be careful and add the measure function. Of course, the $a \rightarrow 0$ limit of Hodge dual has to be modified to cancel the measure function, similar to (5.10). This basis simplifies calculations but does not lead to a big improvement. The measure function in this basis is $\mu(x)=r$.

The method of an auxiliary field $f^{a b}$ introduced in [4] again does not improve a lot. In the sense of the ambiguities of the SW map all three methods are the same as the methods discussed in Section 4. All this suggests that the question of NC gauge theory on a Jordanian $\kappa$-Minkowski needs to be understood better.

\section{Conclusions}

In this paper we demonstrated how one could construct NC gauge theory consistent with deformation of algebra of functions on $\kappa$-Minkowski space-time. Two key ingredients were twist formalism and the Seiberg-Witten map. We used Drinfel'd twist to deform the Hopf algebra of symmetry generators and then used the Hopf algebra action to induce deformation of geometry. This procedure provides differential calculus needed for the construction of a field theory. As a next step, we defined NC gauge transformations. The Seiberg-Witten map insures that these NC gauge transformations are actually induced by the corresponding commutative gauge transformation. Expanding in the deformation parameter led to effective models which could be seen as a possible non-local and non-linear extension of classical electrodynamics. Moreover, we showed that different underlying symmetries, $\operatorname{igl}(1,3)$ and $i w s o(1,3)$, led to two different deformations of the standard theory.

We also described in details the obstacles encountered in our analyses and offered some possible solutions. The failure of the Jordanian twist to provide cyclic integral could be understood as an indication that the underlying symmetry iwso(1,3) and its twisted version are not compatible with the flat metric. The measure we introduced, seemingly ad hoc, might be seen as a consistency requirement. The obstruction we have encountered in the construction of the Hodge dual field-strength tensor is a manifestation of the fact that the introduction of 
a NC geometrical structure prevents decoupling of diffeomorphisms and gauge symmetries. The Hodge dual field-strength tensor includes both gauge and metric degrees of freedom. Consistent $\mathrm{NC}$ deformation of both gauge and geometry imposes that the metric degrees of freedom should transform covariantly under the gauge transformation. This brings in mind the ideas of generalized geometry, a framework in which one organizes and extends the gauge transformations and the diffeomorphisms within $O(d, d)$ group. Applying these ideas in the present context would imply extending our analyses to quasi-Hopf algebras [22, 8, 44] and/or Hopf algebroids [36, 54, 32].

\section{A Appendix}

One can write the symmetrized version of Jordanian twist (which inverse is given in equation (5.1), see also [25]) by taking the formal expansion in the parameter $a$ as:

$$
\begin{aligned}
\mathcal{F}= & 1 \otimes 1+\frac{i a}{2}\left(P_{0} \otimes J-J \otimes P_{0}\right) \\
& +\frac{(i a)^{2}}{4} \frac{1}{2}\left(P_{0}^{2} \otimes J^{2}-2 P_{0} J \otimes J P_{0}-2 J P_{0} \otimes P_{0} J+2 P_{0} J \otimes P_{0} J+J^{2} \otimes P_{0}^{2}\right) \\
& -\frac{(i a)^{3}}{8}\left\{\frac{1}{6} J^{\underline{3}} \otimes P_{0}^{3}-\frac{1}{6} P_{0}^{3} \otimes J^{\underline{3}}+\frac{1}{2} J P_{0}^{2} \otimes J P_{0} J\right. \\
& \left.-\frac{1}{2} J P_{0} J \otimes J P_{0}^{2}+P_{0} J \otimes P_{0}^{2}-P_{0}^{2} \otimes P_{0} J\right\}+\mathcal{O}\left(a^{4}\right),
\end{aligned}
$$

where $J^{\underline{r}}=J(J-1) \cdots(J-r+1), r=1,2, \ldots$ The deformed coproducts for Lorentz and dilatation generators can be also calculated order by order in the deformation parameter $a$ and up to the third order are the following:

$$
\begin{aligned}
\Delta_{\mathrm{up}\left(a^{3}\right)}^{\mathcal{F}}\left(M_{i}\right)= & \Delta_{0}\left(M_{i}\right), \\
\Delta_{\mathrm{up}\left(a^{3}\right)}^{\mathcal{F}}\left(N_{k}\right)= & \Delta_{0}\left(N_{k}\right)+\frac{i a}{2}\left(P_{k} \otimes J-J \otimes P_{k}\right) \\
& -\frac{(i a)^{2}}{4}\left(P_{k} \otimes P_{0} J+P_{0} J \otimes P_{k}+P_{k} P_{0} \otimes J+J \otimes P_{k} P_{0}\right) \\
& +\frac{(i a)^{3}}{8}\left\{P_{k} P_{0}^{2} \otimes J-J \otimes P_{k} P_{0}^{2}+P_{k} P_{0} \otimes P_{0} J-P_{0} J \otimes P_{k} P_{0}\right\}+\mathcal{O}\left(a^{4}\right), \\
\Delta_{\mathrm{up}\left(a^{3}\right)}^{\mathcal{F}}(J)= & \Delta_{0}(J)+\frac{i a}{2}\left(P_{0} \otimes J-J \otimes P_{0}\right) \\
& -\frac{(i a)^{2}}{4}\left(P_{0} \otimes P_{0} J+P_{0} J \otimes P_{0}+P_{0}^{2} \otimes J+J \otimes P_{0}^{2}\right) \\
+ & \frac{(i a)^{3}}{8}\left\{P_{0}^{3} \otimes J-J \otimes P_{0}^{3}-P_{0} J \otimes P_{0}^{2}+P_{0}^{2} \otimes P_{0} J\right\}+\mathcal{O}\left(a^{4}\right) .
\end{aligned}
$$

Here we introduced the following notation for Poincaré algebra generators $M_{i}=\frac{1}{2} \epsilon_{i j k} M_{j k}$ for rotations and $N_{i}=M_{0 i}$ for boosts. The twisted coproduct for momenta written in a compact form (5.2) in Section 5 can be also expanded:

$$
\begin{aligned}
\Delta_{\mathrm{up}\left(a^{3}\right)}^{\mathcal{F}}\left(P_{\mu}\right)= & \Delta_{0}\left(P_{\mu}\right)+\frac{i a}{2}\left(P_{0} \otimes P_{\mu}-P_{\mu} \otimes P_{0}\right)-\frac{(i a)^{2}}{4}\left(P_{0} P_{\mu} \otimes P_{0}+P_{0} \otimes P_{0} P_{\mu}\right) \\
& +\frac{(i a)^{3}}{8}\left\{P_{0} P_{\mu} \otimes P_{0}^{2}-P_{0}^{2} \otimes P_{0} P_{\mu}\right\}+\mathcal{O}\left(a^{4}\right) .
\end{aligned}
$$


Twisted antipodes are the following:

$$
\begin{aligned}
& S^{\mathcal{F}}\left(M_{i}\right)=-M_{i}, \\
& S^{\mathcal{F}}\left(N_{k}\right)=-N_{k}+\frac{i a}{2}\left(P_{k} J-\left(1+\frac{i a}{2} P_{0}\right) J\left(1+\frac{i a}{2} P_{0}\right)^{-1} P_{k}\right), \\
& S^{\mathcal{F}}(J)=-\left(1+\frac{i a}{2} P_{0}\right) J\left(1+\frac{i a}{2} P_{0}\right)^{-1}, \\
& S^{\mathcal{F}}\left(P_{\mu}\right)=-P_{\mu} .
\end{aligned}
$$

To complete the definition of $U_{i w s o(1,3)}^{\mathcal{F}}[[a]]$ we mention that counits stay undeformed:

$$
\epsilon\left(M_{i}\right)=\epsilon\left(N_{k}\right)=\epsilon\left(P_{\mu}\right)=\epsilon(J)=0 .
$$

\section{A.1 Acknowledgements}

The authors are grateful to P. Aschieri for pointing out the reference [25] and to A. Borowiec for discussions. We would also like to thank the anonymous referees for their insightful comments. The work is partially supported by ICTP - SEENET-MTP Project PRJ09 "Cosmology and Strings" in frame of the Southeastern European Network in Theoretical and Mathematical Physics. A.P. acknowledges the financial support of Polish NCN Grant 2011/01/B/ST2/03354. The work of M.D. is supported by Project No. 171031 of the Serbian Ministry of Education and Science.

\section{References}

[1] Agostini A., Amelino-Camelia G., Arzano M., D’Andrea F., Action functional for kappa-Minkowski noncommutative spacetime, hep-th/0407227.

[2] Aschieri P., Castellani L., Noncommutative $D=4$ gravity coupled to fermions, J. High Energy Phys. 2009 (2009), no. 6, 086, 18 pages, arXiv:0902.3817.

[3] Aschieri P., Castellani L., Noncommutative gravity coupled to fermions: second order expansion via SeibergWitten map, J. High Energy Phys. 2012 (2012), no. 7, 184, 27 pages, arXiv:1111.4822.

[4] Aschieri P., Castellani L., Noncommutative gauge fields coupled to noncommutative gravity, Gen. Relativity Gravitation 45 (2013), 581-598, arXiv:1205.1911.

[5] Aschieri P., Dimitrijević M., Meyer F., Wess J., Noncommutative geometry and gravity, Classical Quantum Gravity 23 (2006), 1883-1911, hep-th/0510059.

[6] Ballesteros Á., Bruno N.R., Herranz F.J., A non-commutative Minkowskian spacetime from a quantum AdS algebra, Phys. Lett. B $\mathbf{5 7 4}$ (2003), 276-282, hep-th/0306089.

[7] Ballesteros Á., Herranz F.J., Bruno N.R., Quantum (anti)de Sitter algebras and generalizations of the kappa-Minkowski space, in Proceedings of 11th International Conference on Symmetry Methods in Physics (June 21-24, 2004, Prague), Editors C. Burdik, O. Navratil, S. Posta, Joint Institute for Nuclear Research, Dubna, 2004, 1-20, hep-th/0409295.

[8] Beggs E.J., Majid S., Quantization by cochain twists and nonassociative differentials, J. Math. Phys. 51 (2010), 053522, 32 pages, math.QA/0506450.

[9] Borowiec A., Lukierski J., Pachoł A., Twisting and $\kappa$-Poincaré, arXiv:1312.7807.

[10] Borowiec A., Pachoł A., $\kappa$-Minkowski spacetime as the result of Jordanian twist deformation, Phys. Rev. D 79 (2009), 045012, 11 pages, arXiv:0812.0576.

[11] Bu J.-G., Kim H.-C., Lee Y., Vac C.H., Yee J.H., $\kappa$-deformed spacetime from twist, Phys. Lett. B 665 (2008), 95-99, hep-th/0611175.

[12] Burić M., Latas D., Radovanović V., Trampetić J., Chiral fermions in noncommutative electrodynamics: renormalizability and dispersion, Phys. Rev. D 83 (2011), 045023, 10 pages, arXiv:1009.4603. 
[13] Chatzistavrakidis A., Jonke L., Matrix theory origins of non-geometric fluxes, J. High Energy Phys. 2013 (2013), no. 2, 040, 34 pages, arXiv:1207.6412.

[14] Connes A., Douglas M.R., Schwarz A., Noncommutative geometry and matrix theory: compactification on tori, J. High Energy Phys. 1998 (1998), no. 2, 003, 35 pages, hep-th/9711162.

[15] Daszkiewicz M., Lukierski J., Woronowicz M., $\kappa$-deformed oscillators, the choice of star product and free $\kappa$-deformed quantum fields, J. Phys. A: Math. Theor. 42 (2009), 355201, 18 pages, arXiv:0807.1992.

[16] Dimitrijević M., Jonke L., A twisted look on kappa-Minkowski: U(1) gauge theory, J. High Energy Phys. 2011 (2011), no. 12, 080, 23 pages, arXiv:1107.3475.

[17] Dimitrijević M., Jonke L., Gauge theory on kappa-Minkowski revisited: the twist approach, J. Phys. Conf. Ser. 343 (2012), 012049, 14 pages, arXiv:1110.6767.

[18] Dimitrijević M., Jonke L., Möller L., U(1) gauge field theory on $\kappa$-Minkowski space, J. High Energy Phys. 2005 (2005), no. 9, 068, 15 pages, hep-th/0504129.

[19] Dimitrijević M., Jonke L., Möller L., Tsouchnika E., Wess J., Wohlgenannt M., Deformed field theory on $\kappa$-spacetime, Eur. Phys. J. C Part. Fields 31 (2003), 129-138, hep-th/0307149.

[20] Dimitrijević M., Meyer F., Möller L., Wess J., Gauge theories on the $\kappa$-Minkowski spacetime, Eur. Phys. J. C Part. Fields 36 (2004), 117-126, hep-th/0310116.

[21] Drinfel'd V.G., On constant quasiclassical solutions of the Yang-Baxter equations, Soviet Math. Dokl. 28 (1983), 667-671.

[22] Drinfel'd V.G., Quasi-Hopf algebras, Leningrad Math. J. 1 (1990), 1419-1457.

[23] Felder G., Shoikhet B., Deformation quantization with traces, Lett. Math. Phys. 53 (2000), 75-86, math.QA/0002057.

[24] Gerstenhaber M., Giaquinto A., Schack S.D., Quantum symmetry, in Quantum Groups (Leningrad, 1990), Lecture Notes in Math., Vol. 1510, Springer, Berlin, 1992, 9-46.

[25] Giaquinto A., Zhang J.J., Bialgebra actions, twists, and universal deformation formulas, J. Pure Appl. Algebra 128 (1998), 133-151, hep-th/9411140.

[26] Govindarajan T.R., Gupta K.S., Harikumar E., Meljanac S., Meljanac D., Twisted statistics in $\kappa$-Minkowski spacetime, Phys. Rev. D $\mathbf{7 7}$ (2008), 105010, 6 pages, arXiv:0802.1576.

[27] Hoppe J., Quantum theory of a massless relativistic surface and a two-dimensional bound state problem, Ph.D. Thesis, Massachusetts Institute of Technology, 1982.

[28] Hoppe J., Membranes and matrix models, hep-th/0206192.

[29] Jambor C., Sykora A., Realization of algebras with the help of star-products, hep-th/0405268.

[30] Jurčo B., Möller L., Schraml S., Schupp P., Wess J., Construction of non-abelian gauge theories on noncommutative spaces, Eur. Phys. J. C Part. Fields 21 (2001), 383-388, hep-th/0104153.

[31] Jurčo B., Schraml S., Schupp P., Wess J., Enveloping algebra-valued gauge transformations for non-abelian gauge groups on non-commutative spaces, Eur. Phys. J. C Part. Fields 17 (2000), 521-526, hep-th/0006246.

[32] Jurić T., Kovačević D., Meljanac S., $\kappa$-deformed phase space, Hopf algebroid and twisting, arXiv:1402.0397.

[33] Jurić T., Meljanac S., Štrajn R., Differential forms and $\kappa$-Minkowski spacetime from extended twist, Eur. Phys. J. C $\mathbf{7 3}$ (2013), 2472, 8 pages, arXiv:1211.6612.

[34] Kim H.-C., Lee Y., Rim C., Yee J.H., Differential structure on the $\kappa$-Minkowski spacetime from twist, Phys. Lett. B 671 (2009), 398-401, arXiv:0808.2866.

[35] Kowalski-Glikman J., Introduction to doubly special relativity, in Planck Scale Effects in Astrophysics and Cosmology, Lecture Notes in Phys., Vol. 669, Springer, Berlin, 2005, 131-159, hep-th/0405273.

[36] Lu J.-H., Hopf algebroids and quantum groupoids, Internat. J. Math. 7 (1996), 47-70, q-alg/9505024.

[37] Lukierski J., Nowicki A., Ruegg H., New quantum Poincaré algebra and $\kappa$-deformed field theory, Phys. Lett. B 293 (1992), 344-352.

[38] Lukierski J., Ruegg H., Nowicki A., Tolstoy V.N., q-deformation of Poincaré algebra, Phys. Lett. B 264 (1991), 331-338.

[39] Madore J., The fuzzy sphere, Classical Quantum Gravity 9 (1992), 69-87.

[40] Madore J., An introduction to noncommutative differential geometry and its physical applications, London Mathematical Society Lecture Note Series, Vol. 257, 2nd ed., Cambridge University Press, Cambridge, 1999. 
[41] Meljanac S., Krešić-Jurić S., Differential structure on $\kappa$-Minkowski space, and $\kappa$-Poincaré algebra, Internat. J. Modern Phys. A 26 (2011), 3385-3402, arXiv:1004.4647.

[42] Meljanac S., Samsarov A., Trampetić J., Wohlgenannt M., Scalar field propagation in the $\phi^{4} \kappa$-Minkowski model, J. High Energy Phys. 2011 (2011), no. 12, 010, 23 pages, arXiv:1111.5553.

[43] Möller L., Second order expansion of action functionals of noncommutative gauge theories, J. High Energy Phys. 2004 (2004), no. 10, 063, 20 pages, hep-th/0409085.

[44] Mylonas D., Schupp P., Szabo R.J., Nonassociative geometry and twist deformations in non-geometric string theory, PoS Proc. Sci. (2014), PoS(ICMP2013), 007, 28 pages, arXiv:1402.7306.

[45] Ogievetsky O., Hopf structures on the Borel subalgebra of sl(2), Rend. Circ. Mat. Palermo (2) Suppl. (1994), 185-199.

[46] Ohn C., A *-product on SL(2) and the corresponding nonstandard quantum-U(s)(2)), Lett. Math. Phys. 25 (1992), 85-88.

[47] Schenkel A., Noncommutative gravity and quantum field theory on noncommutative curved spacetimes, Ph.D. Thesis, University of Würzburg, 2011, arXiv:1210.1115.

[48] Schenkel A., Uhlemann C.F., Field theory on curved noncommutative spacetimes, SIGMA 6 (2010), 061, 19 pages, arXiv:1003.3190.

[49] Seiberg N., Witten E., String theory and noncommutative geometry, J. High Energy Phys. 1999 (1999), no. 9, 032, 93 pages, hep-th/9908142.

[50] Sitarz A., Noncommutative differential calculus on the $\kappa$-Minkowski space, Phys. Lett. B 349 (1995), 42-48, hep-th/9409014.

[51] Steinacker H., Noncommutative geometry and matrix models, PoS Proc. Sci. (2011), PoS(QGQGS2011), 004, 27 pages, arXiv:1109.5521.

[52] Tolstoy V.N., Twisted quantum deformations of Lorentz and Poincaré algebras, arXiv:0712.3962.

[53] Ülker K., Yapışkan B., Seiberg-Witten maps to all orders, Phys. Rev. D 77 (2008), 065006, 9 pages, arXiv:0712.0506.

[54] Xu P., Quantum groupoids, Comm. Math. Phys. 216 (2001), 539-581, math.QA/9905192. 